\title{
Coherent transient phenomena in quantum systems by spatially shaping femtosecond optical pulses
}

\author{
Omar El Gawhary, Silvania F. Pereira, and H. Paul Urbach \\ Optics Research Group, Department of Imaging Science and Technology, Delft University of Technology, \\ Lorentzweg 1, NL-2628 CJ Delft, the Netherlands
}

(Received 24 December 2010; published 16 March 2011)

\begin{abstract}
Providing a femtosecond optical pulse with a proper transverse spatial profile represents a fast and relatively simple method to force a quantum system to follow a prescribed temporal evolution. In the present work, we show that the quantum system presents a surprisingly high sensitivity with respect to the spatial shape of the pulse. We discuss an explicit example where differences on the order of a few nanometers in the initial pulse's spot size induce completely different responses in the system under study.
\end{abstract}

DOI: 10.1103/PhysRevA.83.033412

PACS number(s): 32.80.Qk, 42.25.Bs, 42.65.Re

\section{INTRODUCTION}

Visible light plays a privileged role in the investigation of material properties. This is due to the fact that electronic transitions in matter mainly fall in the visible range. This is also the reason that, from the response of a system of interest when excited through an incident electromagnetic field in the visible range, one can get insight about the dynamics of its degrees of freedom and, ultimately, about the structure of matter itself [1,2]. Current research is approaching light-matter interaction from a different viewpoint. In fact, one is no longer interested in only studying how a system responds to an external excitation but also wants to force it to follow specific temporal behaviors. This represents the growing field called coherent quantum control. In particular, an electromagnetic field can be properly tailored in order to induce a certain response on a molecular or atomic system. To achieve this aim, it is necessary that the system interacts with a pulse with a temporal length of the order of hundreds of femtoseconds or shorter. Although the effect of a very short pulse on the transient of a quantum system was discussed already some time ago [3], its realization was limited by the time scale involved and the possibility of generating such short pulses in practice. With the recent advances in femtosecond science, the generation of those pulses has become routine and the control has found application in a great variety of contexts [4-8].

However, in order to do that, the pulse has usually to be modified as function of time. The process is known as pulse shaping. This is usually done by adjusting directly the temporal-frequency spectrum since the pulse's oscillations are too fast to be controlled in time [9]. In this approach, the spatial variation of the pulse is usually neglected. Recently, some works studied possible ways to include spatial shaping in this scenario, with techniques that essentially transform an original pulse in an array of multiple pulses, spatially separated, each endowed with its own time behavior [10,11]. In the present work, we face the issue with a different goal. In fact, our interest is to investigate how different responses of the quantum system can be induced by exclusively manipulating the initial spatial profile of one single pulse. We will show that phenomena like coherent transients or enhanced transition rates, to name a few, can be obtained without difficulty, simply by spatially shaping a single short pulse. However, we will also show that the interaction between a structured pulse and a system presents very high sensitivity with respect to the imposed pulse's spatial size, an appealing feature with possible important implications in metrology and spectroscopy.

The paper is organized as follows. In the next section, we describe the generic formalism for the propagation of a fully vectorial pulsed beam, with a nontrivial transverse spatial profile. Then, in Sec. III, we focus on the special case of a pulse with a Bessel profile and analyze the effect of this profile by discussing the interaction with a specific two-state system. Finally, we summarize the main results in the Conclusions.

\section{PULSE WITH STRUCTURED INPUT TRANSVERSE DISTRIBUTION}

Let us suppose a $O x y z$ Cartesian reference system has been defined in a spatial region of interest. Let $\boldsymbol{E}(x, y, z, t)$ be the electric part of an electromagnetic field, propagating in this region of space. We assume the distribution of the transverse component $\boldsymbol{E}_{\perp}(x, y, 0, t)$ of $\boldsymbol{E}(x, y, z, t)$ on a input plane, $z=0$, say,

$$
\boldsymbol{E}_{\perp}(x, y, 0, t)=[\hat{\mathbf{x}} f(x, y)+\hat{\mathbf{y}} g(x, y)] u(t) .
$$

In Eq. (1), $f(x, y), g(x, y)$ denote the spatial distributions of the $x$ and $y$ components and $u(t)$ is a common temporal evolution on $z=0$. $\hat{\mathbf{x}}, \hat{\mathbf{y}}, \hat{\mathbf{z}}$ indicating the Cartesian unit vectors.

Although the knowledge of $\boldsymbol{E}_{\perp}(x, y, 0, t)$ is actually already sufficient to compute the field everywhere, a $z$ component for the electric field is in general also present. That is a consequence of having a divergence-free field, that is, $\boldsymbol{\nabla} \cdot \boldsymbol{E}=$ 0 . In particular, one can show that

$$
\left.\partial_{z} E_{z}(x, y, z, t)\right|_{z=0}=-\left[\partial_{x} f(x, y)-\partial_{y} g(x, y)\right] u(t) .
$$

We are interested, first of all, in writing the expression of electric field $\boldsymbol{E}_{\perp}(x, y, z, t)$ in every plane $z>0$. One can expand this field in terms of spatial and temporal plane waves, as follows:

$$
\begin{aligned}
\boldsymbol{E}_{\perp}(x, y, 0, t)= & \iiint_{\infty}\left\{\boldsymbol{S}_{\perp}^{(0)}(p, q) T^{(0)}(v)\right. \\
& \times \exp [i 2 \pi(p x+q y-v t)]\} d p d q d v,
\end{aligned}
$$


where

$$
\begin{gathered}
S_{\perp}^{(0)}(p, q)=S_{x}^{(0)}(p, q) \hat{\mathbf{x}}+S_{y}^{(0)}(p, q) \hat{\mathbf{y}}, \\
S_{x}^{(0)}(p, q)=\iint_{\infty} f(x, y) \exp [-i 2 \pi(p x+q y)] d x d y, \\
S_{y}^{(0)}(p, q)=\iint_{\infty} g(x, y) \exp [-i 2 \pi(p x+q y)] d x d y,
\end{gathered}
$$

and

$$
T^{(0)}(v)=\int_{-\infty}^{\infty} u(t) \exp (i 2 \pi v t) d t
$$

and $p, q$ represent the spatial and $v$ the temporal frequencies, respectively.

Because the effect of propagation on a single plane wave is known, once the total spectrum at $z=0$ is available, it is possible to compute the spectrum on a different plane, $z>0$, say, as

$$
\boldsymbol{A}^{(z)}(p, q, v)=\boldsymbol{A}^{(0)}(p, q, v) \exp (i 2 \pi m z)
$$

where $\boldsymbol{A}^{(0)}(p, q, v)=\left[\hat{\mathbf{x}} S_{x}(p, q)+\hat{\mathbf{y}} S_{y}(p, q)+\hat{\mathbf{z}} S_{z}(p, q)\right] T^{(0)}$ (v) is the total spectrum at $z=0$ and $m$ takes two forms in the homogeneous and evanescent part of the spectrum:

$$
\begin{gathered}
m=\sqrt{(v / c)^{2}-\left(p^{2}+q^{2}\right)}, \\
p^{2}+q^{2}<(v / c)^{2} \text { (homogeneous) }
\end{gathered}
$$

and

$$
\begin{gathered}
m=i \sqrt{\left(p^{2}+q^{2}\right)-(v / c)^{2}}, \\
p^{2}+q^{2}>(v / c)^{2}(\text { evanescent }) .
\end{gathered}
$$

Here $c$ denotes the speed of light in a vacuum. It is important to emphasize that Eqs. (8) and (9) are consequences of the separation condition ( $v$ being the temporal frequency in vacuum),

$$
\frac{v^{2}}{c^{2}}=p^{2}+q^{2}+m^{2}
$$

which in turn is a consequence of expanding a generic solution of the wave equation as a collection of plane waves.

Once the complex plane-wave amplitude of all these plane waves is given, on a plane $z$, then the field in any other position and time instant can be easily obtained by inverse Fourier transforming it:

$$
\begin{aligned}
\boldsymbol{E}(x, y, z, t)= & \iiint_{\infty} \boldsymbol{A}^{(z)}(p, q, \nu) \\
& \times \exp [i 2 \pi(p x+q y-v t)] d p d q d \nu .
\end{aligned}
$$

From this, it follows that knowing the spectrum at $z=0$ is enough to calculate the electromagnetic field everywhere within the half space $z>0$. An important remark is that while the total spectrum in $z=0, \boldsymbol{A}^{(0)}(p, q, v)$, is actually separable in the spatial frequencies $p, q$, and the temporal frequency $v$, this is no longer true for the spectrum in $z>0, A^{(z)}(p, q, v)$, because of the presence of the term $\exp (i 2 \pi m z)$ in Eq. (7) that actually mixes the two parts.
From Eqs. (2) and (4), it is straightforward to show that

$$
S_{z}^{(0)}(p, q)=-\frac{\left[p S_{x}^{(0)}(p, q)+q S_{y}^{(0)}(p, q)\right]}{m} .
$$

Hence, once the spectra $S_{\perp}^{(0)}(p, q)$ and $T^{(0)}(v)$ are known, one can obtain the electromagnetic field as

$$
\begin{aligned}
& \boldsymbol{E}_{\perp}(x, y, z, t)=\iint_{\infty} \int_{\infty}\left\{\boldsymbol{S}_{\perp}^{(0)}(p, q) T^{(0)}(v) \exp (i 2 \pi m z)\right. \\
& \times \exp [i 2 \pi(p x+q y-v t)]\} d p d q d v, \\
& E_{z}(x, y, z, t) \\
& =\iiint_{\infty}-\frac{\left[p S_{x}^{(0)}(p, q)+q S_{y}^{(0)}(p, q)\right]}{m} T^{(0)}(v) \\
& \quad \times \exp (i 2 \pi m z) \exp [i 2 \pi(p x+q y-v t)] d p d q d v .
\end{aligned}
$$

Equations (13a) and (13b) make explicit the effect of a spatial profile, amplitude, and phase, over the time behavior of a field, and vice versa.

Let us suppose now that one is interested in getting a given time behavior in a specific point $x, y, z$. This can be obtained in two different ways. One method could be to directly impose the desired time behavior by tailoring the signal in time. The problem with this technique is that in optics field oscillations are so fast that this is almost impossible in practice. One can circumvent the problem by working in the Fourier domain. This is the common way pulse shaping has been performed so far, and important results were obtained using this approach [4]. In particular, the possibility of controlling a quantum system has been proven both theoretically and experimentally. However, as we already wrote in the introduction, almost all works based on this method neglect the spatial dependence of the involved electromagnetic fields. Light pulses have the trait of beams, in other words, of fields localized in space around a given mean direction of propagation. Although in many cases the spatial profile is trivial enough that it does not play any important role, in other situations it can be fundamental. In fact, from what we have said so far, once the time evolution $u(t)$ on $z=0$ is given, one can try to design a proper spatial profile in order to induce a determined desired behavior on $x, y, z$. The aim of the next section is to provide a concrete example of how that is possible by discussing the interaction of a two-state system with a specific field.

\section{INTERACTION OF A STRUCTURED PULSED BEAM AND A TWO-STATE SYSTEM: THE CASE OF VECTORIAL BESSEL BEAMS}

In the present section, we will consider an electromagnetic field with a specific $\boldsymbol{E}_{\perp}(x, y, 0, t)$ value. With reference to the notation introduced in the previous section, we choose

$$
\begin{aligned}
& f(x, y)=J_{0}\left(\beta r_{\perp}\right), \\
& g(x, y)=J_{0}\left(\beta r_{\perp}\right),
\end{aligned}
$$

where $r_{\perp}=\sqrt{x^{2}+y^{2}}$. For the time being, we still leave the functional form of $u(t)$ unspecified. In Eqs. (14), $J_{0}$ indicates the zeroth-order Bessel function of first type; $\beta$ is the spatial 
frequency that controls the effective beam size since the radius of the beam, $r_{0}$, can be considered roughly equal to $\beta / 2.4$, that is, in correspondence to the first zero of the $J_{0}$ function. Monochromatic Bessel beams are the best known examples of nondiffracting beams and can nowadays be generated in the laboratory using different methods [12-15]. Strictly speaking, they are not physically realizable, because their total energy in plane $z$ constant is infinite, but in practice the introduction of an envelope removes this problem, as in the known case of Bessel-Gauss beams [16,17]. The nondiffracting nature of these fields stems from noticing that all plane waves, constituting the angular spectrum, possess the same phase velocity along the $z$ direction and thus interfere always in the same way during propagation. It follows that the field changes during propagation only by a trivial phase factor, while the amplitude remains the same. In terms of the angular spectra $S_{x}^{(0)}(p, q)$ and $S_{y}^{(0)}(p, q)$, this can be mathematically expressed in the form

$$
\begin{aligned}
& S_{x}^{(0)}(p, q)=\frac{\delta\left(\varrho-\frac{\beta}{2 \pi}\right)}{\beta}, \\
& S_{y}^{(0)}(p, q)=\frac{\delta\left(\varrho-\frac{\beta}{2 \pi}\right)}{\beta},
\end{aligned}
$$

where $\varrho=\sqrt{p^{2}+q^{2}}$ and $\delta(\xi)$ is the Dirac function of argument $\xi$. Since the two components on $z=0$ have equal amplitude and are always in phase, the transverse field $\boldsymbol{E}_{\perp}$ is linearly polarized. Later on, in the appendix, the case of fields endowed with nonuniform polarization through the whole $x, y$ plane is also considered. In case of a Bessel-Gauss beam, the input distribution takes the expression

$$
J_{0}\left(\beta r_{\perp}\right) \exp \left(-r_{\perp}^{2} / w_{0}^{2}\right),
$$

with $w_{0}$ as the waist of the Gaussian. However, the presence of the Gaussian modulation affects the spectrum of the original Bessel field. Interestingly, the closed-form expression for such spectrum is available and reads [18]

$$
\pi w_{0}^{2} \exp \left(-\beta^{2} w_{0}^{2} / 4\right) \exp \left(-\pi^{2} w_{0}^{2} \rho^{2}\right) I_{0}\left(\pi \beta w_{0}^{2} \rho\right),
$$

where $I_{0}$ is the zeroth-order modified Bessel function of the first kind. This spectrum tends, with good accuracy, to those in Eqs. (15) when the product $\beta w_{0}$ is sufficiently large (larger than 10, let us say) [19].

Within this assumption, using the circular coordinates in spatial Fourier space, and inserting in Eqs. (13) the spectra of Eqs. (15), we obtain

$$
\begin{aligned}
& E_{\alpha}(x, y, z, t) \\
& =J_{0}\left(\beta r_{\perp}\right) \int_{\nu} T^{(0)}(\nu) \exp \left[i 2 \pi \sqrt{v^{2} / c^{2}-\beta^{2} /\left(4 \pi^{2}\right)} z\right] \\
& \quad \times \exp (-i 2 \pi v t) d \nu
\end{aligned}
$$

with $\alpha=x, y$.

The advantage of using a Bessel field as input distribution derives from the fact that, due to the particular form of the spatial spectra $S_{x}^{(0)}(p, q)$ and $S_{y}^{(0)}(p, q)$ [Eqs. (15)], the dependence on the transverse coordinate $r_{\perp}$ is the same in any plane where $z=$ constant, and, additionally, the temporal behavior depends on the initial transverse spatial profile by means of the parameter $\beta$ only. In fact, Eq. (18) shows that the field in any plane $z$ can be written as the product of a factor depending on the transverse spatial coordinate only and a factor depending on time $t$ and $z$. In case of other field distributions, the effect of space on time would be less simple. For instance, in case of an input Gaussian distribution, it would not be possible to reduce the expression for the propagated field in the same simple form as in Eq. (18a), where the transverse spatial dependence is multiplied by the $z$-temporal one. Hence, using a nondiffracting field as input spatial modulation for the original pulse partly disentangles spatial effects from temporal ones, making the subsequent analysis much easier.

Coming back to the electric field, one can write the expression for the $z$ component as well, which results in

$$
\begin{aligned}
E_{z}(x, y, z, t)= & \int_{-\infty}^{\infty} \int_{\infty}^{\infty} \int_{\infty}^{\infty}-\frac{1}{\sqrt{v^{2} / c^{2}-\left(p^{2}+q^{2}\right)}} \\
& \times\left[p S_{x}^{(0)}(p, q)+q S_{y}^{(0)}(p, q)\right] T^{(0)}(v) \\
& \times \exp [i 2 \pi(p x+q y-v t)] \\
& \times \exp (i 2 \pi m z) d p d q d v \\
= & \int_{-\infty}^{\infty} \int_{0}^{\infty} \int_{0}^{2 \pi}-\frac{1}{\sqrt{v^{2} / c^{2}-\varrho^{2}}} \\
& \times\left[\varrho \cos \varphi S_{x}^{(0)}(\varrho, \varphi)+\varrho \sin \varphi S_{y}^{(0)}(\varrho, \varphi)\right] T^{(0)}(v) \\
& \times \exp \left[i 2 \pi \varrho r_{\perp} \cos (\varphi-\vartheta)\right] \exp (-i 2 \pi v t) \\
& \times \exp (i 2 \pi m z) \varrho d \varrho d \varphi d \nu,
\end{aligned}
$$

where the circular coordinates in real and Fourier domain were defined as follows:

$$
\begin{aligned}
& x=r_{\perp} \cos \vartheta, \\
& y=r_{\perp} \sin \vartheta,
\end{aligned}
$$

and

$$
\begin{aligned}
& p=\varrho \cos \varphi, \\
& q=\varrho \sin \varphi,
\end{aligned}
$$

with $r_{\perp}, \varrho \in[0, \infty)$ and $\vartheta, \varphi \in[0,2 \pi)$. On substituting the spectra in Eq. (15) into Eq. (19), one gets

$$
\begin{aligned}
E_{z}(x, y, z, t)= & -i \frac{\beta}{2 \pi} J_{1}\left(\beta r_{\perp}\right)(\cos \vartheta+\sin \vartheta) \\
& \times \int_{-\infty}^{\infty} \frac{1}{\sqrt{v^{2} / c^{2}-\beta^{2} /\left(4 \pi^{2}\right)}} T^{(0)}(\nu) \\
& \times \exp \left[i 2 \pi \sqrt{v^{2} / c^{2}-\beta^{2} /\left(4 \pi^{2}\right)} z\right] \\
& \times \exp (-i 2 \pi v t) d \nu
\end{aligned}
$$

where $J_{1}\left(\beta r_{\perp}\right)$ is the first-order Bessel function of first type. From this equation, we see that while the transverse components have a maximum on the $z$ axis, the $z$ component has always a zero exactly there because of the presence of a phase singularity.

So far, we have simply described the propagation in free space of an electromagnetic pulse with assigned space and time dependence on an starting plane $z=0$. We now turn our attention to how this field interacts with a quantum system located somewhere in the half space $z>0$. Our aim is to show how different the system response can be, after being excited by the field, just by changing the field spot size on the $z=0$ 
plane. The field-matter interaction is described in the dipole model approximation.

\section{A. Dipole model}

Let us assume, from now on, that a quantum system, being an ensemble of atoms or molecules, occupies a region of space around the point $(0,0, z)$. Since the system spatial sizes are usually much smaller than any optical field spot size, we will consider only the incident field on the $z$ axis. This is equivalent to saying that, from a spatial point of view, the field is actually seen as a uniform plane wave in any $x, y$ plane. The quantum system is described as a two-state system, $|g\rangle$ and $|e\rangle$, with energy levels $E_{g}$ and $E_{e}$, respectively. When the external field is present, the system is in a state $|\Psi\rangle$, which is a linear combination of the two possible states

$|\Psi\rangle=c_{g}(t) \exp \left(-i E_{g} t / \hbar\right)|g\rangle+c_{e}(t) \exp \left(-i E_{e} t / \hbar\right)|e\rangle$,

where $\hbar=h /(2 \pi)$, with $h$ being the Planck's constant.

In the framework of the first-order perturbation theory of quantum mechanics, the time evolution of the coefficients $c_{e}(t)$, related to the transition probability from $|g\rangle$ to $|e\rangle$, can be written as

$$
c_{e}(t)=\frac{1}{i \hbar} \int_{-\infty}^{t} H_{e g}^{\prime} \exp \left(i 2 \pi v_{e g} t^{\prime}\right) d t^{\prime},
$$

where it is assumed that in a remote past the system was initially in the state $|g\rangle$. In Eq. (24) $v_{e g}=\left(E_{e}-E_{g}\right) /(2 \pi \hbar)$ is the transition frequency and $H_{e g}^{\prime}=\left\langle e\left|H^{\prime}\right| g\right\rangle$ is the matrix element from $|g\rangle$ to $|e\rangle$. In the dipole approximation, we have $H^{\prime}=-Q \mathbf{r} \cdot \boldsymbol{E}(x, y, z, t)$, where $Q$ is the electric charge of the dipole and $\boldsymbol{r}=(x, y, z)$. Since only the field on $z$-axis will be considered, Eq. (24) can be rewritten as

$$
c_{e}(t)=\frac{-Q}{i \hbar} \int_{-\infty}^{t}\left\langle e\left|\mathbf{r} \cdot \boldsymbol{E}\left(0,0, z, t^{\prime}\right)\right| g\right\rangle \exp \left(i 2 \pi v_{e g} t^{\prime}\right) d t^{\prime} .
$$

The dipole is supposed to be located at $(0,0, z)$ and oscillating along the $x$ axis. Under these hypotheses, Eq. (25) becomes

$$
c_{e}(t)=\frac{M_{e g}}{i \hbar} \int_{-\infty}^{t} E_{x}\left(0,0, z, t^{\prime}\right) \exp \left(i 2 \pi v_{e g} t^{\prime}\right) d t^{\prime},
$$

where $M_{e g}=-Q\langle e|x| g\rangle$ is the dipole moment matrix element. As to the function $u(t)$ appearing in Eq. (6), the following expression will be assumed:

$$
u(t)=\exp \left(-t^{2} / \tau^{2}\right) \cos \left(2 \pi v_{0} t\right),
$$

with $\tau$ representing the pulse length and $v_{0}$ representing the carrier. It follows that, within the present model, the system-field interaction is completely determined once $M_{e g}$, $\nu_{e g}, \beta, \tau$, and $\nu_{0}$ are specified. From now on, we take $\nu_{0}=$ $v_{e g}=1.998616 \times 10^{14} \mathrm{~Hz}$ (corresponding to a wavelength in vacuum of $\lambda_{0}=1.5 \mu \mathrm{m}$ ) and $\tau=100 \mathrm{fs}$, and in our analysis we will consider the interaction for different choices of $\beta$. Our interest is to show that only by adjusting the field spot size, on $z=0$, can completely different dynamics be induced in the quantum system.
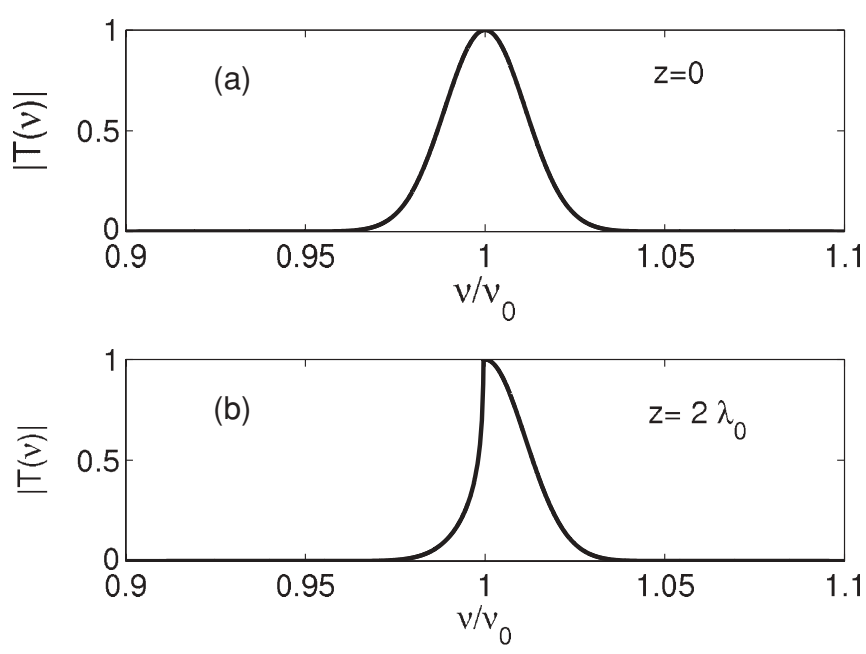

FIG. 1. Behavior of the normalized spectrum $\left|T^{(0)}(v) \exp \left[i 2 \pi \sqrt{v^{2} / c^{2}-\beta^{2} /\left(4 \pi^{2}\right)} z\right]\right|$, when the spatial distribution on $z=0$ is a zeroth-order Bessel function of first type, for $z=0$ (subplot a) and $z=2 \lambda_{0}$ (subplot b). $v_{0}=c / \lambda_{0}$. In the plot, we have chosen $\lambda_{0}=1.5 \mu \mathrm{m}, \beta=2 \pi \nu_{c} / c$, where $\nu_{c}=0.9995 v_{0}$ is the cutoff frequency.

\section{B. Transition rates}

In all cases we assume that the interaction will take place far from the input plane. In other words, the quantum system is located at a position $(0,0, z)$ with $z \gg \lambda_{0}$.

Three main cases will be considered. First, we start by considering a pulse that at $z=0$ has no spatial structure; that is, when in Eqs. (18)-(22), we set $\beta=0$. This is the case usually treated in the literature. In practice, it deals with an uniform plane wave, in space, modulated by a Gaussian envelope, in time, on $z=0$.

In the other two cases, the Bessel profile is considered, and we choose $\beta=\beta_{1}=2 \pi v_{1} / c$ and $\beta=\beta_{2}=2 \pi v_{2} / c$ respectively. Here, $v_{1}=v_{e g} / 1.0005$ and $\nu_{2}=v_{e g} / 0.9995$ represent two different cutoff frequencies. In fact, from Eqs. (18) and (19), one sees that once a spatial profile is imposed, on $z=0$, some of the plane waves constituting the pulse can fall in the evanescent part of the field spectrum, being, in this way, suppressed under free space propagation. For the reader's convenience, we report in Fig. 1 the effect of propagation on the spectrum $T^{(0)}(v) \exp \left[i 2 \pi \sqrt{v^{2} / c^{2}-\beta^{2} /\left(4 \pi^{2}\right)} z\right]$. Figure 1(a) is evaluated at $z=0$ while Fig. $1(\mathrm{~b})$ is evaluated at $z=2 \lambda_{0}$. In the plots, the cutoff frequency is chosen to be $0.9995 v_{0}$, where $v_{0}=c / \lambda_{0}$ is the carrier. However, as we are going to show soon, it makes a big difference, from a system point of view, whether its resonance frequency, $v_{e g}$, resides in the homogeneous or evanescent region of that spectrum. In particular, when $v_{1}=v_{e g} / 1.0005$ the transition frequency $v_{e g}$ is actually carried by the field, while when $v_{2}=v_{e g} / 0.9995$ it belongs to that part of the spectrum that is under the cutoff and is not able to reach the far zone.

Figure 2(a) shows the corresponding transition rates in Eq. (26) for the three cases when the system is positioned at $\left(0,0,20 \lambda_{0}\right)$. The continuous curve represents the $\beta=0$ case (as we said, a simple transform-limited pulse) where the system undergoes a rapid transition, on a time scale of about $100 \mathrm{fs}$, and then reaches the final state. In particular, for large 
time delays (longer than the pulse duration), the transition probability depends only on the energy content at frequency $v_{g e}$ contained in the incident field. In contrast, the dotted curve represents the transition rate of the same identical system, at position $z$, when excited by the pulse field having $\beta=\beta_{1}$. As is clear from the plot, the transient shows a completely different behavior, tending, in any case to the same asymptotic value. This is due to the fact that the long time limit depends only on the amplitude of the spectral component at $v_{e g}$ frequency, that is actually carried by the field. One should also note that in some time instants the transition rate is enhanced with respect to the case of just using a transform-limited pulse $(\beta=0)$. This effect is purely determined by the input spatial modulation. The oscillations around the asymptotic value are a confirmation that a real coherent transient is taking place. Additionally, it was elsewhere recognized already that this enhancement is produced by a change of the destructive interference occurring between two frequency components, belonging to the field spectrum, in symmetrical position with respect to $v_{e g}$ [6]. In this case, the spatial shaping does not turn such an interference into a constructive one but just unbalances the two contributions in order to reduce the effect of destructive interference.

Finally, the third curve shows the same response of the same system when $\beta=\beta_{2}$. In this case, the resonance frequency is under the cutoff (i.e., all corresponding plane waves are evanescent). Because the system is far enough from the input plane, there is no component at $v_{e g}$ frequency. This means that the long time delay value must be zero, as is shown in the plot. Additionally, although there are residual oscillations, proving that coherent phenomena take place, the transition rate is notably damped if compared to the previous two cases. For completeness, in the subplots $\mathrm{b}$ and $\mathrm{c}$ we also report the evolution in the phase space $\left(\operatorname{Re}\left\{c_{e}(t)\right\}, \operatorname{Im}\left\{c_{e}(t)\right\}\right)(\operatorname{Re}$ and $\operatorname{Im}$ stand for real and imaginary part) from the initial state toward the final one, only for the cases where $\beta$ is different from zero.

It is very interesting to notice that such a variety of behaviors is only induced by changing the parameter $\beta$ that in turn determines the beam size. More important, the system shows an extremely high sensitivity with respect to such beam size, which represents a central result of the present work. For instance, the different transients shown in Fig. 2 (dashed and dotted curves) correspond to a beam diameter of $1.1464 \mu \mathrm{m}\left(\beta=\beta_{1}\right)$ and $1.1453 \mu \mathrm{m}\left(\beta=\beta_{2}\right)$, respectively. The difference between the two is only $1.1 \mathrm{~nm}$. We can justify this surprisingly high sensitivity with a simple argument. First of all, we estimate the beam's radius by looking at the position of the first zero in the Bessel function $J_{0}\left(\beta r_{\perp}\right)$, which is located, as we said before, at $r_{0} \cong 2.4 / \beta$. In our example, we have chosen $\beta=2 \pi v_{c} / c$, where by $v_{c}$ indicates the cutoff frequency; $r_{0}$ is related to $v_{c}$ through the relation

$$
r_{0} \cong \frac{2.4 c}{2 \pi v_{c}} .
$$

The sensitivity of $v_{c}$ with respect to $r_{0}$ is then

$$
\frac{\partial v_{c}}{\partial r_{0}} \cong\left|\frac{2.4 c}{2 \pi r_{0}^{2}}\right|
$$
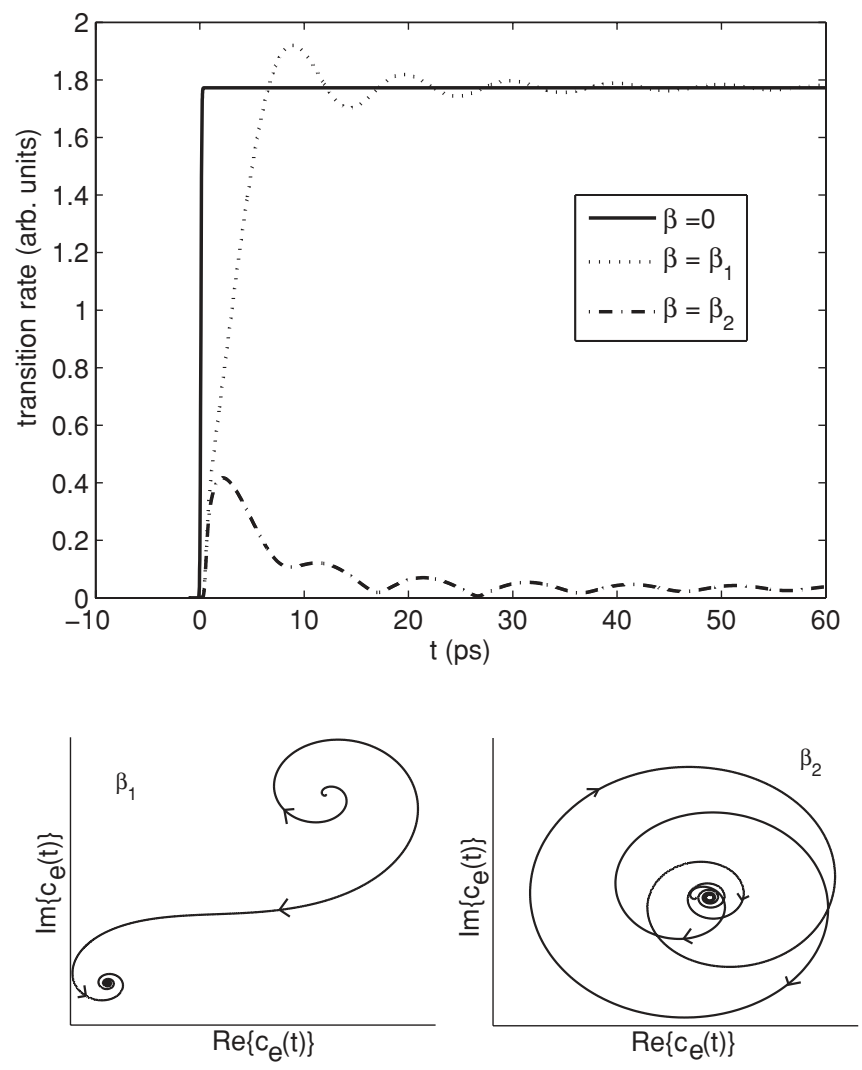

FIG. 2. Transition rates induced in an two-state system by a pulse with a Bessel spatial profile for different values of the field spot size on the $z=0$ plane. In panel (a), the absolute value of the transition rate $c_{e}(t)$ as a function of time is shown. The solid line in represents a diffraction limited pulse $(\beta=0)$, with uniform spatial distribution on $z=0$. The dotted line describes the case when $\beta=\beta_{1}=2 \pi v_{1} / c$, with $v_{1}=v_{e g} / 1.0005$. The line-dotted line refers to the third case, when $\beta=\beta_{2}=2 \pi v_{2} / c$ with $\nu_{2}=v_{e g} / 0.9995$. The interaction with the quantum system is supposed to take place on $z=20 \lambda_{0}$, where $\lambda_{0}=1.5 \mu \mathrm{m}$. Panels (b) and (c) show the evolution in phase space for the cases of $\beta_{1}$ and $\beta_{2}$, respectively.

From Eq. (29), one sees that around the value of $1 \mu \mathrm{m}$ for the beam's diameter $\left(2 r_{0}\right)$, the sensitivity becomes $2.29024 \times$ $10^{14} \mathrm{~Hz} / \mathrm{m}$. Since the response of the system strongly depends on whether its resonance frequency $v_{e g}$ is under cutoff, this high sensitivity shows that in principle even a few nanometers change in the beam size can be enough to go from one situation ( $v_{e g}$ under cutoff) to the other ( $v_{e g}$ above cutoff). However, it is important to realize that this does not imply that a control of the beam size on a scale of nanometers is necessary in order to obtain the described effects, because the marked difference in situations occurs for any pair of frequencies of which one is above and the other below cutoff. In any practical implementation, it would suffice to choose two different beams, with different spot sizes (i.e., two different values of $\beta$ ), such that the corresponding cutoff frequencies would be one bigger and the other smaller than $v_{e g}$. In any case, this strong dependence on the initial spatial size of the beam, around the cutoff frequency, is an appealing property that makes the proposed method interesting for future possible 
applications such as, among others, in selective excitation of small emitters like nanoparticles.

\section{CONCLUSIONS}

We have discussed the role that a nontrivial spatial modulation can play in the field of quantum control. In particular, we showed how one can induce completely different behaviors in a quantum system just by changing the transverse spatial size of a pulse of interest, even in vacuum. We provided the general mathematical descriptions, and we focused on a particular case where the spatial distribution of the pulse, on the starting plane, is a Bessel function. Under the assumption that the quantum system is modeled by means of a two-state model, we derived the transition rate associated to a particular spatial size. We also discussed the sensitivity of the whole process on the input beam size. As we showed, since the frequency cutoff occurring during propagation, caused by the imposed spatial shape, gets really sharp a few wavelengths from the input plane (in the sense clarified in the previous sections), the excitation of the system is strongly affected by change in the field's diameter of even a few nanometers.

\section{ACKNOWLEDGMENTS}

The authors acknowledge Nandini Bhattacharya and Mounir Zeitouny for extensive discussions and comments on the manuscript. O. El Gawhary acknowledges the Surface Physics for Advanced Manufacturing European Project, within the FP7 Marie Curie Research and Initial Training Network Program, for funding the research under Project No. 215723.

\section{APPENDIX: SHAPING THE INPUT FIELD TO EXCITE DIPOLES IN OTHER DIRECTIONS}

In Sec. III, we have described the interaction of a femtosecond pulse, endowed with a Bessel lateral profile, and an atomic system, modeled as a two-state system, positioned on the $z$ axis. For the interaction, the standard dipole approximation has been used and, additionally, the assumption that only the field on axis was seen by the quantum system. With the choice made in Eq. (14), we had a $z$ field component with a zero on $z=0$, which did not allow interaction with dipoles oriented along the $z$ direction. The aim of the present section is to describe a more general case, where the input electric field on $z=0$ has a nonuniform polarization state, and to show how that actually flips the field on axis, in order to interact with a dipole oriented along $z$, too. Also, this is an occasion to generalize the formalism to fields with linear, but nonuniform, polarization states.

With reference to Eq. (1), we take now

$$
\begin{aligned}
& f(x, y)=J_{m}\left(\beta r_{\perp}\right) \cos (m \vartheta+\alpha), \\
& g(x, y)=J_{m}\left(\beta r_{\perp}\right) \sin (m \vartheta+\alpha),
\end{aligned}
$$

where $J_{m}(\cdot)$ denotes a $m$ th-order Bessel function of first kind, $\alpha \in[0,2 \pi)$ is a constant angle, and $r_{\perp}$ and $\theta$ are defined in Eq. (20). In particular, the transverse field $\boldsymbol{E}_{\perp}$ is still linearly polarized in every point on the $x, y$ plane, but with a polarization direction that depends on the angular variable $\vartheta$ and forms, in any point, an angle $\alpha$ with the radial direction. Optical, monochromatic beams with such a state of polarization were studied recently in the paraxial framework [20] as well as their free-space behavior in the nonparaxial regime or when focused by high numerical aperture optical systems $[21,22]$. From a practical viewpoint, these types of fields can be generated using a spatial light modulator that shapes the amplitude and phase at the same time, or by special classes of laser sources, like a concentric-circle-grating, surface-emitting (CCGSE) semiconductor laser [23,24]. It is of some interest to derive the structure of the field's lines on $z=0$, that are determined by the equation

$$
\frac{d y}{d x}=\frac{E_{y}}{E_{x}}=\tan (m \vartheta) .
$$

This equation can be rewritten in polar coordinates and gives

$$
\frac{\sin \vartheta d r_{\perp}+r_{\perp} \cos \vartheta d r_{\perp}}{\cos \vartheta d r_{\perp}-r_{\perp} \sin \vartheta d r_{\perp}}=\tan (m \vartheta) .
$$

After simple algebra, Eq. (A3) yields

$$
\frac{d r_{\perp}}{r_{\perp}}=d \vartheta \frac{\cos [(m-1) \vartheta+\alpha]}{\sin [(m-1) \vartheta+\alpha]} .
$$

To get the shape of field lines, we have to distinguish two cases: $m \neq 1$ and $m=1$. When $m \neq 1$, the field's lines become

$$
r_{\perp}=R\left|\sin [(m-1) \vartheta+\alpha]^{1 /(m-1)}\right|,
$$

with $R$ a constant. To show how these fields' lines look, we plot in Fig. 3 the case when $m=4$ and $\alpha=\pi / 4$. The shape reminds us of a daisy, with the number of petals $2(m-1)$ depending on the integer $m$. The angle $\alpha$ represents just an offset: Varying it leads to an overall rotation of the daisy. The other case corresponds to choosing $m=1$. Equation (A4) then

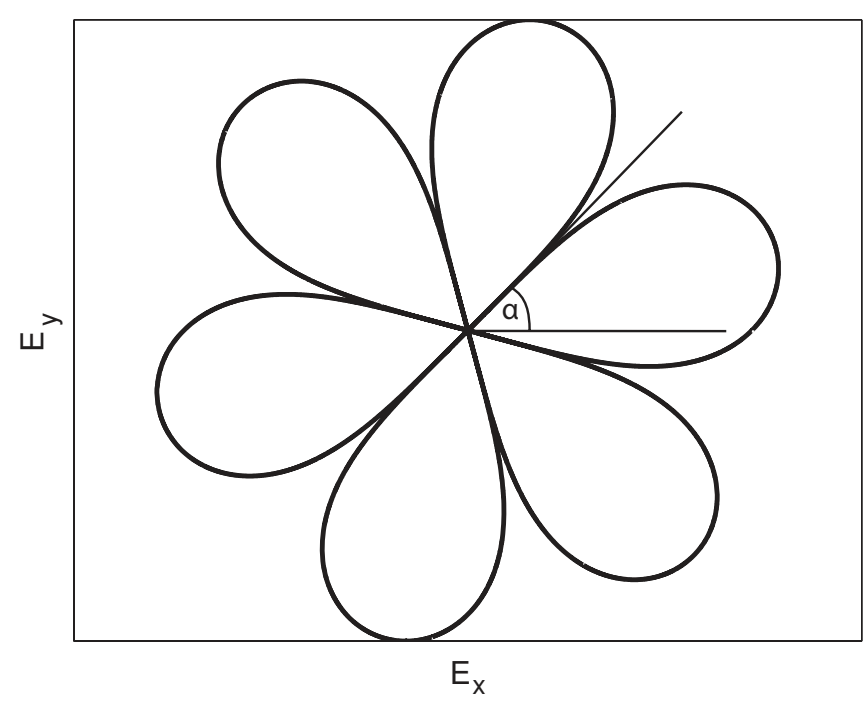

FIG. 3. Field's lines, $r_{\perp}=R\left|\sin [(m-1) \vartheta+\alpha]^{1 /(m-1)}\right|$, when the field components are $f(x, y)=J_{m}\left(\beta r_{\perp}\right) \cos (m \vartheta+\alpha)$ and $g(x, y)=J_{m}\left(\beta r_{\perp}\right) \sin (m \vartheta+\alpha)$, respectively [see Eqs. (A1)]. The figure was obtained by choosing $m=4$ and $\alpha=\pi / 4$. Considered the special properties of nondiffracting beams, such polarization state is invariant during free-space propagation. 
leads to the following form for the field's lines:

$$
r_{\perp}=R \exp \left(\frac{\vartheta}{\tan \alpha}\right),
$$

which is the expression of a logarithmic spiral. It is very interesting that such polarization states are invariant during propagation. In fact, one has for $E_{x}$ and $E_{y}$

$$
\begin{aligned}
E_{x}(x, y, z, t)= & i^{m} J_{m}\left(\beta r_{\perp}\right) \cos (m \vartheta+\alpha) \int_{-\infty}^{\infty} T^{(0)}(v) \\
& \times \exp \left[i 2 \pi \sqrt{v^{2} / c^{2}-\beta^{2} /\left(4 \pi^{2}\right)} z\right] \\
& \times \exp (-i 2 \pi v t) d v \\
E_{y}(x, y, z, t)= & i^{m} J_{m}\left(\beta r_{\perp}\right) \sin (m \vartheta+\alpha) \int_{-\infty}^{\infty} T^{(0)}(v) \\
& \times \exp \left[i 2 \pi \sqrt{v^{2} / c^{2}-\beta^{2} /\left(4 \pi^{2}\right)} z\right] \\
& \times \exp (-i 2 \pi v t) d v
\end{aligned}
$$

fulfilling exactly the same equation [Eq. (A2)] for all values of $z$. As to the $z$ component, $E_{z}$, after simple, but lengthy, algebra one obtains

$$
\begin{aligned}
E_{z}(x, y, z, t)= & -\frac{1}{2}\left\{i^{(m+1)} J_{m+1}\left(\beta r_{\perp}\right) \cos [(m+1) \vartheta+\alpha]\right. \\
& +i^{(m-1)} J_{m-1}\left(\beta r_{\perp}\right) \cos [(m-1) \vartheta+\alpha] \\
& +i^{m} J_{m+1}\left(\beta r_{\perp}\right) \sin [(m+1) \vartheta+\alpha] \\
& \left.-i^{m} J_{m-1}\left(\beta r_{\perp}\right) \sin [(m-1) \vartheta+\alpha]\right\} \\
& \times \int_{-\infty}^{\infty} \frac{1}{\sqrt{v^{2} / c^{2}-\beta^{2} /\left(4 \pi^{2}\right)}} T^{(0)}(v) \\
& \times \exp \left[i 2 \pi \sqrt{v^{2} / c^{2}-\beta^{2} /\left(4 \pi^{2}\right)} z\right] \\
& \times \exp (-i 2 \pi v t) d v
\end{aligned}
$$

Let us focus on one specific case, for instance when $m=1$. Equation (A8) reduces to

$$
\begin{aligned}
E_{z}(x, y, z, t)= & -\frac{1}{2}\left\{-J_{2}\left(\beta r_{\perp}\right) \cos (2 \vartheta+\alpha)+J_{0}\left(\beta r_{\perp}\right) \cos \alpha\right. \\
& \left.+i J_{2}\left(\beta r_{\perp}\right) \sin (2 \vartheta+\alpha)-i J_{0}\left(\beta r_{\perp}\right) \sin \alpha\right\} \\
& \times \int_{-\infty}^{\infty} \frac{1}{\sqrt{v^{2} / c^{2}-\beta^{2} /\left(4 \pi^{2}\right)}} T^{(0)}(v) \\
& \times \exp \left[i 2 \pi \sqrt{v^{2} / c^{2}-\beta^{2} /\left(4 \pi^{2}\right)} z\right] \\
& \times \exp (-i 2 \pi v t) d v .
\end{aligned}
$$

The presence of the phase vortex in the transverse components $E_{x}$ and $E_{y}$ has two effects. On one hand, it creates a phase singularity on $z=0$ for $E_{x}$ and $E_{y}$. Hence, these two fields must vanish everywhere along the $z$ axis during propagation. In fact, as it is easy to show, they take the expressions (recall that $\alpha=x, y$ )

$$
\begin{aligned}
E_{x}(x, y, z, t)= & i J_{1}\left(\beta r_{\perp}\right) \cos (\vartheta+\alpha) \int_{-\infty}^{\infty} T^{(0)}(v) \\
& \times \exp \left[i 2 \pi \sqrt{v^{2} / c^{2}-\beta^{2} /\left(4 \pi^{2}\right)} z\right] \\
& \times \exp (-i 2 \pi v t) d \nu, \\
E_{y}(x, y, z, t)= & i J_{1}\left(\beta r_{\perp}\right) \sin (\vartheta+\alpha) \int_{-\infty}^{\infty} T^{(0)}(v) \\
& \times \exp \left[i 2 \pi \sqrt{v^{2} / c^{2}-\beta^{2} /\left(4 \pi^{2}\right)} z\right] \\
& \times \exp (-i 2 \pi v t) d \nu,
\end{aligned}
$$

and they vanish when $r_{\perp}=0$, as they should. On the other hand, since the total electric field is divergence free, the third component, $E_{z}$, has to be different from zero on the same axis. The presence of such $z$ component on the axis allows the excitations of dipole oriented along $z$ as well, which is what we wanted to show in this appendix.
[1] B. H. Brandsen and C. J. Joachain, Physics of Atoms and Molecules (Essex, Prentice Hall, 2003).

[2] J. J. Sakurai, Modern Quantum Mechanics (Reading, AddisonWesley, 1994).

[3] D. J. Tannor and S. A. Rice, J. Chem. Phys. 83, 5013 (1985).

[4] Femtosecond Laser Spectroscopy, Peter Hannaford Ed. (Boston, Springer, 2005).

[5] S. Zamith, J. Degert, S. Stock, B. de Beauvoir, V. Blanchet, M. A. Bouchene, and B. Girard, Phys. Rev. Lett. 87, 033001 (2001).

[6] N. Dudovich, D. Oron, and Y. Silberberg, Phys. Rev. Lett. 88, 123004 (2002).

[7] F. E. Zimmer, A. Andre, M. D. Lukin, and M. Fleischhauer, Optics Commun. 264, 441 (2006).

[8] D. Oron and Y. Silberberg, Opt. Express 13, 9903 (2005).

[9] J. W. Goodman, Introduction to Fourier Optics, 3rd ed. (Roberts and Company Publishers, 2005).

[10] M. M. Wefers, K. A. Nelson, and A. M. Weiner, Opt. Lett. 21, 746 (1996).

[11] R. M. Koehl, T. Hattori, and K. A. Nelson, Optics Commun. 157, 57 (1998).
[12] J. Durnin, J. J. Miceli, and J. H. Eberly, Phys. Rev. Lett. 58, 1499 (1987).

[13] V. Garces-Chavez, D. McGloin, H. Melville, W. Sibbett, and K. Dholakia, Nature (London) 419, 145 (2002).

[14] J. Arlt, V. Garces-Chavez, W. Sibbett, and K. Dholakia, Optics Commun. 197, 239 (2001).

[15] S. N. Kurilkina, A. A. Ryzhevich, S. B. Bushuk, and S. V. Solonevich, Quantum Electron. 38, 349 (2008).

[16] F. Gori, G. Guattari, and C. Padovani, Optics Commun. 6, 491 (1987).

[17] O. El Gawhary and S. Severini, J. Opt. A: Pure Appl. Opt. 8, 409 (2006).

[18] R. Borghi, and M. Santarsiero, Opt. Lett. 22, 262 (1997).

[19] O. El Gawhary and S. Severini, Opt. Lett. 33, 1360 (2008).

[20] F. Gori, J. Opt. Soc. Am. A 38, 1612 (2001).

[21] R. Borghi and M. Santarsiero, J. Opt. Soc. Am. A 21, 2029 (2004).

[22] R. Borghi and M. Santarsiero, J. Opt. Soc. Am. A 22, 1420 (2005)

[23] A. A. Tovar and G. H. Clark, J. Opt. Soc. Am. A 14, 3333 (1997).

[24] R. H. Jordan, D. G. Hall, O. King, G. Wicks, and S. Rishton, J. Opt. Soc. Am. A 14, 449 (1997). 